\title{
Cartografía de índices indirectos de manifestaciones de hidrocarburos en el Golfo de Guacanayabo, Cuba
}

\author{
Moraima Álvarez-Ortiz ${ }^{1} \mathbb{0}$; Ernesto Leyva-Ramos ${ }^{1} \mathbb{D}$; Willy Roberto Rodríguez-Miranda ${ }^{2 *} \mathbb{C}$ \\ Forma de citar: Álvarez-Ortiz; M.; Leyva-Ramos, E.; Rodríguez-Miranda, W.R. (2021). Cartografía de índices \\ indirectos de manifestaciones de hidrocarburos en el Golfo de Guacanayabo, Cuba. Boletín de Geología, 43(2), \\ 79-93. https://doi.org/10.18273/revbol.v43n2-2021005
}

\begin{abstract}
Resumen
La necesidad de disponer de reservas gaso-petrolíferas es imperiosa en el mundo contemporáneo y en el caso de Cuba la situación se acentúa debido a factores externos. Por esta razón numerosas investigaciones son realizadas en el territorio nacional para localizar estructuras favorables donde puedan encontrase acumulaciones de hidrocarburos. El objetivo de esta investigación ha sido estudiar los sedimentos marinos del Golfo de Guacanayabo (Cuba Oriental), los indicios de hidrocarburos en los fondos marinos y el establecimiento de las áreas de mayor interés. Para este estudio se ha combinado la batimetría y la sísmica de reflexión de alta resolución para realizar la cartografía de estructuras anómalas clasificadas como posibles pockmarks y volcanes de lodo (“mud volcanoes”), morfologías generadas por emisiones naturales de hidrocarburos en los fondos marinos. Asimismo, se ha establecido la relación entre emanaciones gaseosas y presencia de gas en los sedimentos del fondo marino y las estructuras profundas y someras en la región. Finalmente, se han establecido las áreas con mayores potenciales a partir de la integración de todos los resultados disponibles. Este estudio permite asegurar las potencialidades del Golfo de Guacanayabo para estudios posteriores en relación con la presencia de hidrocarburos en el subsuelo.
\end{abstract}

Palabras clave: Batimetría; Pockmarks; Hidrocarburos; Sísmica; Volcanes de lodos.

\section{Mapping of indirect indices of hydrocarbon manifestations in the Gulf of Guacanayabo, Cuba}

\begin{abstract}
The need to have gas and oil reserves is imperative in the contemporary world and in the case of Cuba the situation is accentuated due to external factors. For this reason, numerous investigations are carried out in the national territory to locate favorable structures where accumulations of hydrocarbons can be found. The objective of this research has been to study the marine sediments of the Gulf of Guacanayabo (Eastern Cuba), the indications of hydrocarbons in the seabed and the establishment of the areas of greatest interest. For this study, the combined use of bathymetry and high-resolution reflection seismic was used to map anomalous structures classified as possible pockmarks and mud volcanoes, morphologies generated by natural hydrocarbon emissions in the seabed. Likewise, the relationship between gaseous emanations and the presence of gas in the sediments of the seabed and the deep and shallow structures in the region has been established. Finally, the areas with the greatest potential have been established from the integration of all the available results. This study allows to ensure the potentialities of the Gulf of Guacanayabo for subsequent studies in relation to the presence of hydrocarbons in the subsoil.
\end{abstract}

Keywords: Bathymetry; Pockmarks; Hydrocarbons; Seismic; Mud volcanoes.

${ }^{1}$ Geocuba Estudios Marinos, Regla, La Habana, Cuba. mora@emarinos.geocuba.cu; ernesto@emarinos.geocuba.cu ${ }^{2}$ Universidad Tecnológica de La Habana “José Antonio Echeverría”, Departamento de Geociencias, Marianao, La Habana, Cuba. (*)willy@civil.cujae.edu.cu 


\section{Introducción}

La existencia de cráteres o depresiones en el fondo marino como consecuencia de escapes de fluidos del subsuelo (gases o líquidos), son conocidos como pockmarks por su denominación inglesa. Estas depresiones se encuentran en los mares de cualquier latitud y también en algunos lagos, son en general de forma circular, alargada e irregular con diámetros que pueden alcanzar desde unidades de metros hasta 10 a 12 $\mathrm{km}$. Se pueden presentar aisladas, agrupadas o alineadas en cadenas que puedan llegar a tener $50 \mathrm{~km}$ de largo. En general se asocian a la presencia de gas, hidratos de metano o petróleo (Krämer et al., 2017; Ceramicola et al., 2018; Spina, 2018; Audsley et al., 2019).

El mecanismo de formación de los pockmarks se basa en el arrastre de sedimentos que provoca la emisión de fluidos del subsuelo, los que al descargarse en la columna de agua se distribuyen según las corrientes creando un déficit de masa que induce el hundimiento del fondo marino. Aparecen en los registros sísmicos como una hendidura vertical en forma de "V" con una sucesión en los reflectores sísmicos infra-yacentes (Hovland et al., 2002).

Un volcán de lodo es considerado como una geoforma que tiene una expresión topográfica definida (Ceramicola et al., 2018). Están formados por el gas que escapa hacia la superficie del fondo marino cuando encuentra un conducto que generalmente es una falla o fractura. Este flujo de gas viene acompañado por material de poca cohesión, agua, salmueras y lodo de más baja densidad que las secuencias sedimentarias adyacentes, por lo que es típicamente reconocido como un intervalo de baja velocidad representado por facies sísmicas transparentes, de bajas amplitudes (Spina, 2017).

Según Rejas-Alejos (2009), la presencia y distribución de los volcanes de lodo están fuertemente controladas por factores geológicos (grandes acumulaciones de material terrígeno de 8 a $22 \mathrm{~km}$ de espesor, presencia de capas con comportamiento plástico situadas en profundidad, acumulaciones de gas en profundidad), factores tectónicos (procesos de subsidencia rápidos debidos a la gran tasa de acumulación de sedimentos, presencia de diapiros o anticlinales, presencia de fallas, actividad sísmica, procesos isostáticos), factores geoquímicos (generación de petróleo en profundidad, deshidratación de minerales arcillosos) y factores hidrogeológicos (circulación de fluidos a través de fracturas).
Las primeras estructuras geológicas que revelaron indicios de manifestaciones de hidrocarburos mediante la aplicación de métodos geofísicos marinos fueron detectadas en la década del 60 del siglo XX, durante una investigación con sonar de barrido lateral que permitió descubrir depresiones anómalas del fondo marino denominadas pockmarks (King y MacLean, 1970).

En los registros sísmicos los pockmarks se reflejan como depresiones del sub-fondo marino reflejando una sucesión vertical de las fases de la señal sísmica, y los volcanes de lodo como ruptura e irrupción de los reflectores sísmicos dentro de una unidad de facies sísmicas con reflectores continuos lateralmente y reflexiones difusas o transparentes debajo. Además, pueden ser identificadas emanaciones gaseosas a partir de patrones asociados con reflexiones opacas, apantallamientos acústicos, cortinas acústicas, turbidez acústica y columnas acústicas.

En los últimos años la literatura especializada reporta importantes estudios aplicando técnicas geofísicas marinas de alta resolución para la búsqueda de pockmarks y volcanes de lodo como índices indirectos de presencia de hidrocarburos (García-Gil et al., 2000).

En La Ría de Vigo al noroeste de España, en el año 1999, se realizó un estudio con perfiles geofísicos de alta resolución (sísmica, sonar de barrido lateral y batimetría) que permitió detectar y caracterizar la presencia de gas en los sedimentos y en la columna de agua. Los investigadores clasificaron en cuatro tipos diferentes las acumulaciones de gas basándose en los caracteres de sus respuestas sísmicas, geometría y dimensiones, las cuales fueron apantallamientos acústicos, cortinas acústicas, turbidez acústica y columna acústica, y establecieron tres tipos de evidencias de escape de gas que fueron las plumas acústicas, las nubes acústicas y los pockmarks. Las distintas formas de acumulación de gas descritas podrían estar relacionadas con el porcentaje de gas contenido en los sedimentos, siendo mayor en los apantallamientos acústicos y cortinas acústicas, que en los otros tipos de acumulaciones. El hecho de que ese gas se acumule o escape estará controlado por la efectividad del sello supra-yacente, destacándose, por consiguiente, la importancia de las facies sedimentarias como factor de control, tanto vertical como lateral de la presencia de gas en La Ría de Vigo (García-Gil et al., 1999). 
A partir de investigaciones realizadas con métodos sísmicos de alta resolución y batimetría en el Golfo de Cádiz, con una profundidad de estudio entre 200 y $1200 \mathrm{~m}$, se detectaron numerosas morfologías en forma de volcanes de lodos y pockmarks relacionados con la emisión masiva de hidrocarburos (Somoza et al., 2002, 2003). La presencia de columnas de gas atrapado en estos sedimentos del fondo marino genera anomalías acústicas ("gas chimmeys”) resultado de la absorción de gran parte de la energía (Somoza et al., 2012). También se observaron valores anómalos que se reflejaron en los perfiles sísmicos como reflexiones o ecos puntuales identificados en la columna de agua hasta unos $50 \mathrm{~m}$ sobre el fondo, causados por diferentes impedancias acústicas presentes en las burbujas de gas y de agua. Estas anomalías se identificaron sobre el talud superior del Golfo de Cádiz, concretamente encima del área ocupada por los pockmarks superficiales y valles ciegos ("blind valleys"), lo cual indica que el gas está actualmente escapando del sedimento marino (Ercilla y Baraza, 1996; León et al., 2010).

En el margen continental de África Occidental, en la cuenca del río Congo, con profundidades del mar entre 950 y $1900 \mathrm{~m}$, a partir de los resultados de un levantamiento sísmico 3D de alta resolución, de un levantamiento geoquímico y otras técnicas, se detectó la existencia de gas termo-génico y petróleo en los sedimentos del fondo de los pockmarks del área de estudio. También los métodos sísmicos detectaron una capa de $300 \mathrm{~m}$ de espesor de hidratos de metano, por debajo de la cual se encontró una acumulación de gas (Gay et al., 2006).

En el Océano Índico, en las Islas Maldivas, existen depresiones del fondo marino, con diámetros de hasta $3000 \mathrm{~m}$ y relieve que puede alcanzar $180 \mathrm{~m}$, en una zona donde la profundidad del mar es de $300 \mathrm{~m}$ aproximadamente. Estos cráteres han sido interpretados como pockmarks formados a partir de la salida de gas y otros fluidos a la superficie del fondo marino. Según los resultados de la adquisición sísmica multicanal se pudo determinar la relación de las depresiones del fondo con estructuras ubicadas en profundidad, ya que bajo las depresiones o pockmarks fueron detectados puntos brillantes y disminución de la frecuencia instantánea, hechos que han sido asociados a lentes de gas, pues también han sido observadas las características propias de las chimeneas de gas (Betzler et al., 2011).

En los trabajos realizados por Quintero-Ramírez (2012) se aborda el tema de la interpretación sísmica de volcanes de lodo en la zona occidental del abanico del delta del río Magdalena, en el Caribe Colombiano. Aquí se observó que las anomalías sísmicas presentaban características similares, como la forma de cono y ausencia de facies en profundidad por debajo de esta estructura, donde se observa un carácter sísmico caótico con blanqueamiento en muchas zonas. En este trabajo se definieron tres clases de volcanes de lodo con base en el mecanismo que los genera: ascenso de lodo a través de fallas o fracturas desde capas sedimentarias, material lodoso que escapa al fondo marino por medio de fracturas ubicadas en crestas de pliegues anticlinales y diapiros de lodo comunicados con el fondo marino a través de algunas fracturas.

En Cuba, durante los años 1987 y 1988, se realizaron investigaciones geofísicas en la Bahía de Cárdenas y áreas adyacentes para conocer las características fundamentales del fondo marino de la región y de las rocas subyacentes. El mapa de anomalías de los patrones de reflexión obtenido de los registros sísmicos y batimétricos mostró una serie de estructuras sismo-geológicas bien definidas, observándose levantamientos y hundimientos de bloques. Además, se observaron reflexiones opacas en diferentes intervalos de los registros sísmicos con un patrón posiblemente asociado con emanaciones gaseosas (Álvarez-Ortiz et al., 2007).

También en Cuba, durante el año 2010, a partir de imágenes satelitales que cubrían desde el sur de Cienfuegos hasta el Golfo de Ana María, se han observado doce anomalías en el fondo marino que parecen ser hundimientos o depresiones, de forma casi circular y de gran tamaño (2000 metros de diámetro), que guardan mucha similitud con los denominados pockmarks. Se analizaron líneas sísmicas 2D y se pudo distinguir en una de las líneas sísmicas, para tiempos menores que $0,5 \mathrm{~s}$, varios indicios asociados a la presencia de gas, como falta de continuidad en los reflectores, aparición de una reflexión fuerte y extendida que puede ser asociada a un punto brillante "flat spot" y el abombamiento de varios reflectores (Morales-Carrillo et al., 2014).

A partir de estos antecedentes, este estudio, realizado con datos batimétricos y perfiles sísmicos de alta resolución, tiene el objetivo de cartografiar estructuras de los fondos marinos del Golfo de Guacanayabo que indiquen indicios de emisiones de hidrocarburos en el subsuelo. 
Características geológicas y geomorfológicas del área de estudio

El Golfo de Guacanayabo se encuentra ubicado en la parte sur-oriental de la isla de Cuba, entre las provincias de Granma, Camagüey, Las Tunas y el archipiélago de los Jardines de la Reina (Figura 1). En sus aguas desembocan ríos de gran importancia regional y nacional como el Cauto, Najasa, Tana y el Yara. Se caracteriza por tener gran número de cayos y arrecifes coralinos en sus aguas poco profundas, propiciando la población de diversas especies biológicas que constituyen una riqueza marina fundamental en la explotación pesquera cubana.

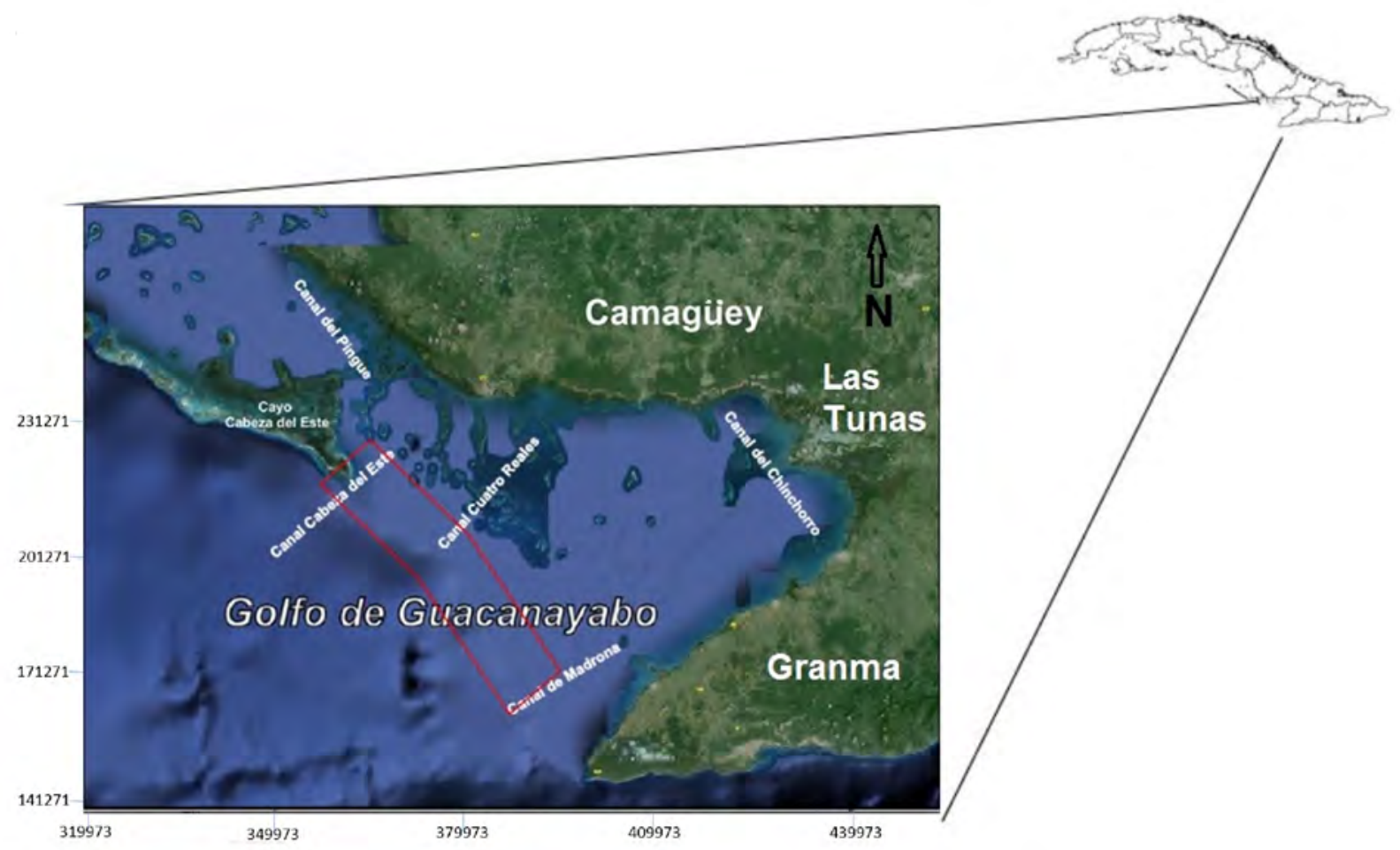

Figura 1. Ubicación geográfica del Golfo de Guacanayabo donde se muestra el área de estudio con el polígono en rojo.

Los fondos de las zonas costeras y el sistema lagunar de la región son de tipo fangoso, aunque en algunas franjas de Manzanillo, Las Coloradas y Cabo Cruz existen playas de arenas blancas y fondos transparentes. El relieve submarino es irregular con suaves pendientes, se destaca la precedencia de bajos cayos y bancos surcados por canalizos estrechos y profundos, así como el desarrollo de arrecifes coralinos en el talud insular. Estos mares son pocos profundos alcanzando solo los 20 y $22 \mathrm{~m}$ de calado en la laguna interior (IturraldeVinent y García-Casco, 2011).

Desde el punto de vista geológico el área de estudio pertenece a la cuenca Cauto-Guacanayabo y está situado sobre la zona de la falla Cauto-Nipe de dirección NE-SO, donde se desarrolla el delta del río
Cauto. En la cuenca domina una alta capacidad de acomodo de la carga de sedimentos con tendencia a la no compensación o colmatación, al parecer un proceso de larga data, posiblemente desde el Cretácico tardío al Eoceno (Iturralde-Vinent y García-Casco, 2011).

La cuenca Cauto-Guacanayabo se extiende sobre la llanura del Cauto y el Golfo de Guacanayabo. La región se asocia con una depresión alargada en dirección latitudinal cuyo borde norte está dado por un sistema de fallas normales lístricas que provocan el hundimiento escalonado de la cuenca hacia su eje, ligeramente desplazado hacia el sur de la misma. Se reconoce como una cuenca de retro-arco del Cretácico, aunque no existen testigos de rocas de esta edad que hayan sido recuperados durante las perforaciones 
realizadas, sin embargo, la existencia de una gran anomalía regional negativa del campo gravitacional no puede ser explicada únicamente por los paquetes de rocas terciarias aun cuando su espesor llegue a ser del orden de 5,0 km en algunos lugares. La presencia de elementos de reflexión coherentes, aunque difíciles de correlacionar, hasta tiempos iguales o mayores a $3,0 \mathrm{~s}$, es un factor por el cual se supone la existencia por debajo del Terciario de rocas sedimentarias, presumiblemente del Cretácico, entre 2,0 y 4,0 km de espesor (Álvarez-Castro et al., 2007).

Durante el Cretácico Superior (CampanianoMaastrichtiano) se activó la falla transcurrente Cauto-Nipe para dejar emplazado el territorio de Cuba Oriental - La Española (COE). El régimen transtensional de la cuenca de apertura del Cauto se mantuvo activo de forma significativa durante los finales del Cretácico Superior y más intensamente en el Paleógeno. El tope de las rocas del Cretácico se hundió considerablemente en el eje de extensión de la depresión y ahora hay sectores donde sobrepasa los 5,0 $\mathrm{km}$ de profundidad. En la misma época en que ocurrió lo anterior, al sur del Golfo de Guacanayabo sucedía una compresión, asociada a la transcurrencia, que dio lugar a la formación de estructuras plegadas.

En levantamientos geológicos realizados en la zona de Jardines de la Reina han sido encontradas muestras de petróleo en las playas de los cayos Caguama, Paloma, y Rabihorcado, estos crudos no han sufrido alteración por biodegradación y se presentaron como muestras frescas con apenas cierto nivel de evaporación, bajo contenido de azufre (0,3\%), aparentemente generados por rocas madre de origen marino - deltaico o fluvio - deltaicas con alto contenido de material vegetal de edad Cretácico Superior o más jóvenes (ÁlvarezCastro et al., 2007).

\section{Materiales y métodos}

Para la adquisición de los datos sísmicos fue empleado el sistema sísmico de reflexión de la firma "APPLIED ACOUSTICS". Durante los trabajos se utilizaron un rango de frecuencias entre $800 \mathrm{~Hz}$ y $2400 \mathrm{kHz}$, con una distancia entre el emisor y el receptor de 2,0 m, una potencia de $500 \mathrm{~J}$ y una extensión de barrido de $125 \mathrm{~m} / \mathrm{s}$, obteniéndose una relación resolución/ penetración adecuada en las capas del subsuelo. Los datos obtenidos fueron almacenados en un sistema geofísico integrado, de adquisición y procesamiento digital (Álvarez-Ortiz et al., 2017).

El procesamiento de los datos geofísicos se realizó mediante el software SurveyEngineSeismic + con el objetivo de detectar las anomalías acústicas presentes en los registros sísmicos y con ello la posible presencia de hidrocarburos en los sedimentos marinos del Golfo de Guacanayabo. Se utilizó un conjunto de herramientas que incluyó filtros de frecuencia, filtros de oleaje, rectificación y ganancia variable en el tiempo lo que permitió obtener imágenes de muy buena calidad para su posterior interpretación, como se muestra en la Figura 2.

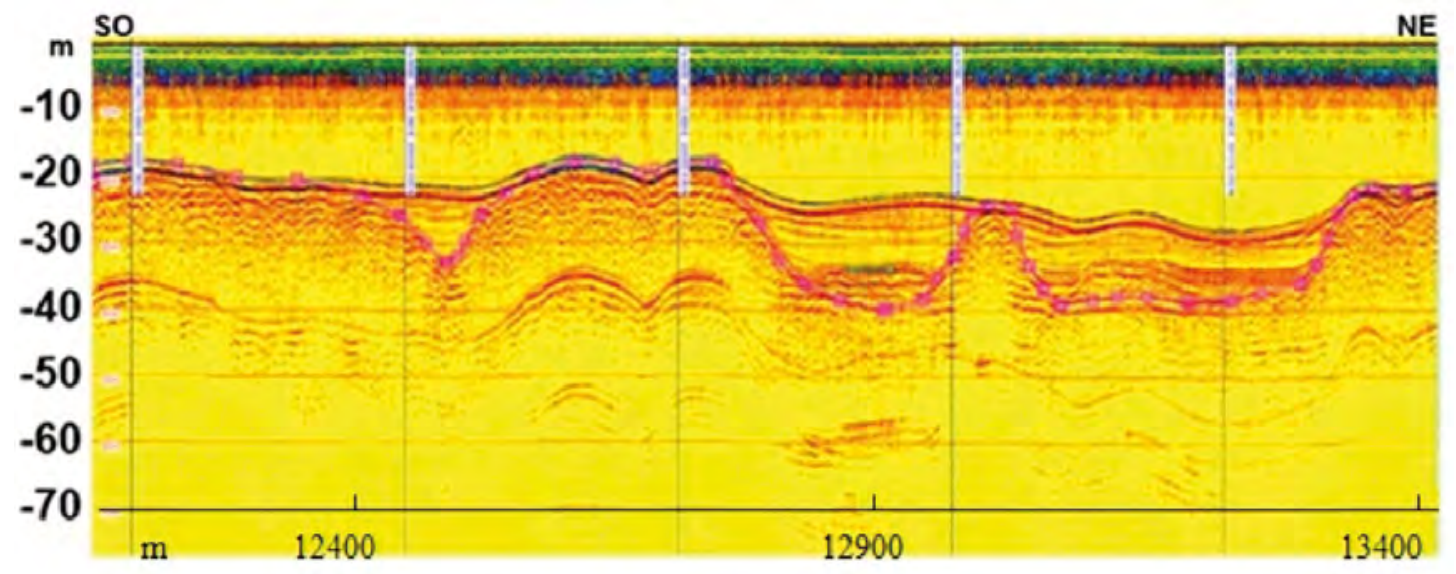

Figura 2. Registro sísmico de alta resolución de la región del Golfo de Guacanayabo. 
Los perfiles de sondeo batimétrico se realizaron simultáneos con los de la sísmica de reflexión por lo que se emplearon las mismas condiciones y sistema de navegación.

Para garantizar la base altimétrica las profundidades fueron referidas al nivel medio del mar (NMM), se determinaron las correcciones por marea con el empleo de una estación provisional de medición en la zona de estudio. Las observaciones de marea se realizaron de forma ininterrumpida durante la realización del Sondeo Batimétrico. El mareógrafo fue comprobado diariamente por altura y hora.

El levantamiento batimétrico del Golfo de Guacanayabo se ejecutó con el sistema de ecosondas Stonex formado por una computadora y un transductor, donde se registra una sola frecuencia de $200 \mathrm{kHz}$ y la resolución en la determinación de las profundidades es de 0,01 m. La precisión en la determinación de la profundidad es 0,01 $\mathrm{m} \pm 0,1 \%$ (Leyva-Ramos, 2017).

El procesamiento final de los datos digitales obtenidos en los registros de campo fue realizado en el software Surfer, donde se realizaron las correcciones necesarias, se regularizaron los datos y confeccionaron los mapas y perfiles batimétricos correspondientes al sector de estudio. El software QGIS se ha utilizado para representar el mapa batimétrico.

\section{Resultados}

\section{Evidencias sísmicas de emisiones de hidrocarburos}

Las concentraciones gaseosas, al tener una impedancia acústica muy diferente a la del medio reflector, tienden a absorber la energía de la onda acústica emitida, creando en los perfiles sísmicos un patrón acústico que puede ser claramente detectado. Estos patrones son denominados apantallamientos acústicos, cortinas acústicas, turbidez acústica o columnas acústicas que por lo general están asociados a la filtración, migración o concentración de emanaciones gaseosas en los sedimentos marinos.

El procesamiento de los registros sísmicos permitió identificar depresiones superficiales o enterradas en el fondo marino, reflejando una sucesión vertical en el caso de los denominados pockmarks, como se presenta en la Figura 3.

Las morfologías en forma de cono asociadas a los volcanes de lodos, aparecieron en el área de estudio como una irrupción de los reflectores sísmicos elevándose las fronteras en el registro y presentando en profundidad paquetes de facies transparentes, amplitudes bajas o carácter caótico (Figura 4).

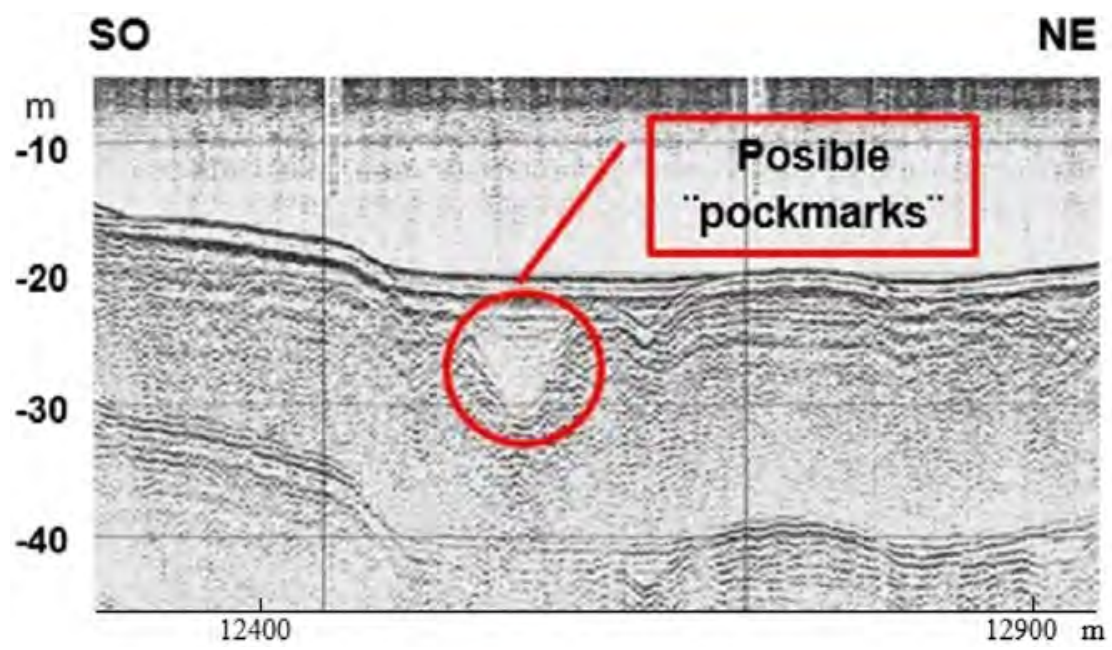

Figura 3. Registro sísmico en profundidad con posible presencia de un posible pockmarks enterrado. 


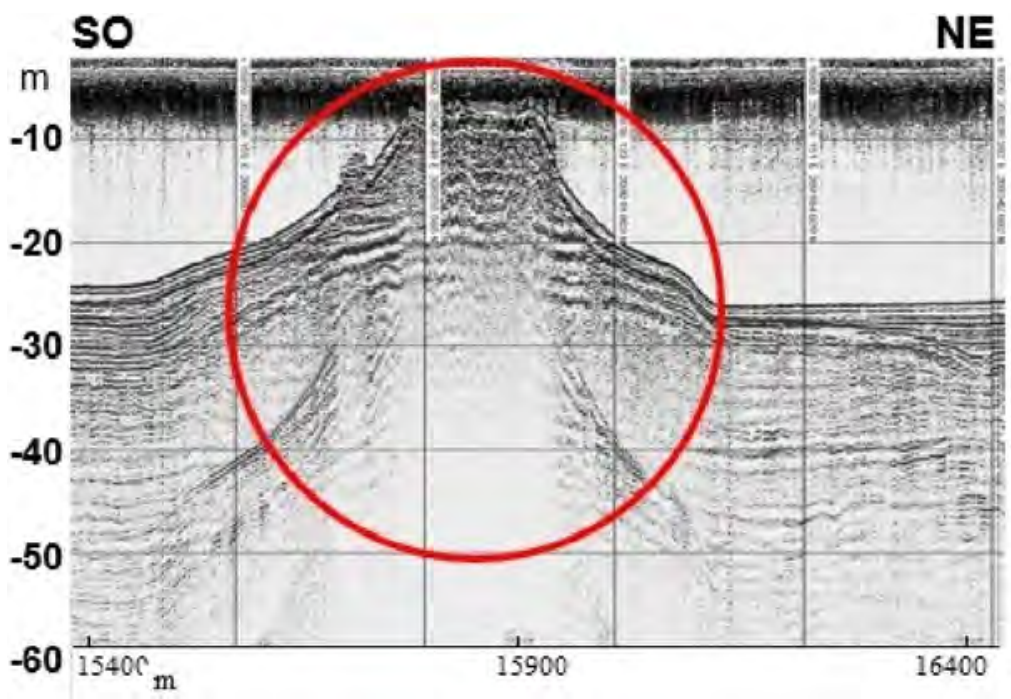

Figura 4. Perfil de sísmica de reflexión de alta resolución de un posible volcán de lodo. Notar las emisiones laterales relacionadas con montículos submarinos.

La batimetría se empleó para determinar las profundidades del lecho marino en el área de estudio, apoyar la interpretación de los registros sismoacústicos, garantizar la base altimétrica y contribuir a la detección de anomalías superficiales asociadas a la búsqueda de índices indirectos de presencia de hidrocarburos en el Golfo de Guacanayabo.

\section{Morfologías submarinas}

El mapa batimétrico de la región permite realizar un análisis más detallado de las morfologías en forma de cráter (pockmarks) y en forma de cono asociadas a volcanes de lodo (Figura 5).

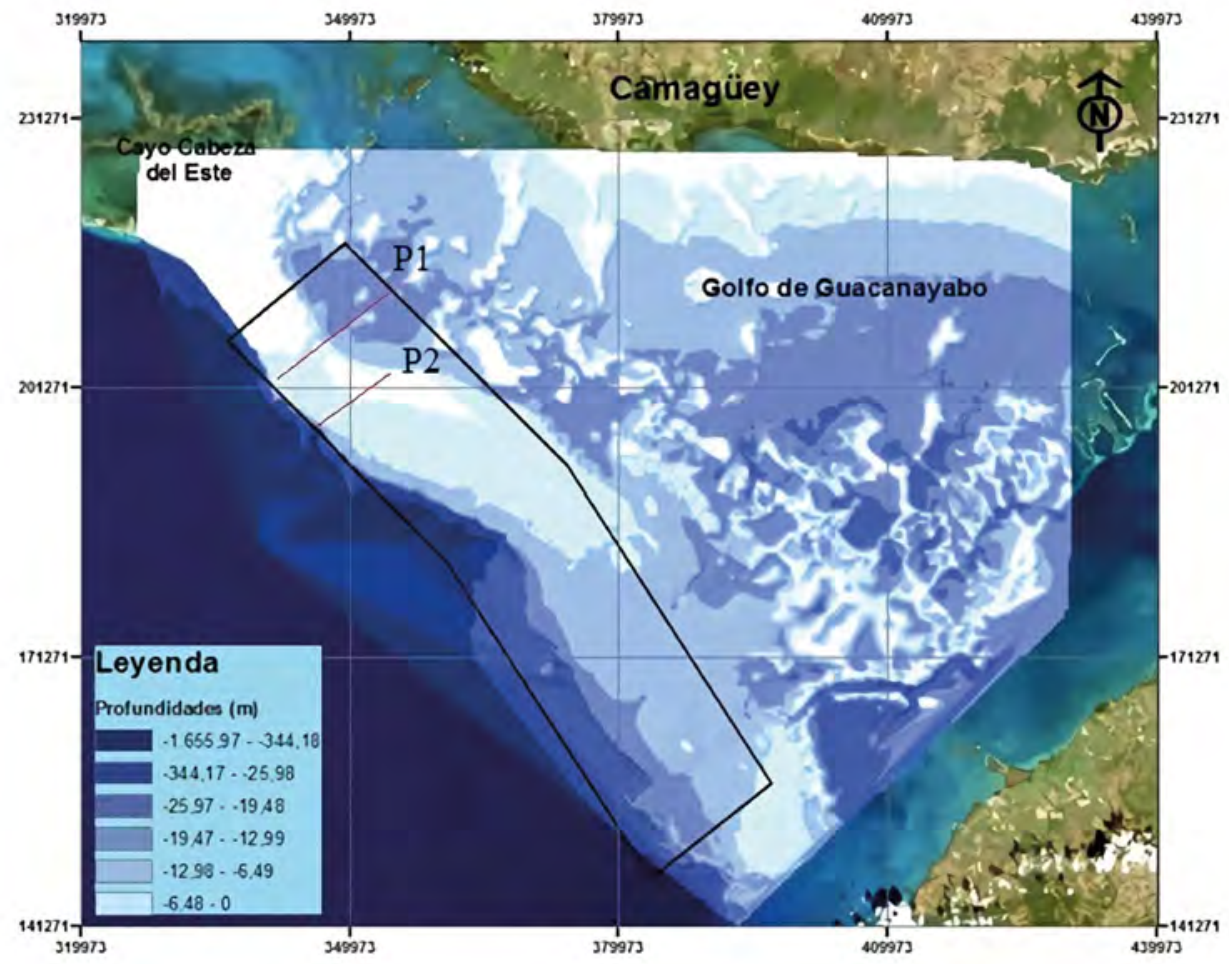

Figura 5. Batimetría del área de estudio, se señalan los perfiles 1 y 2 , donde aparecen posibles volcanes de lodo y pockmarks. 
La Figura 6 muestra los perfiles batimétricos 1 y 2 en los que se detecta la presencia de un volcán de lodo y tres posibles pockmarks.

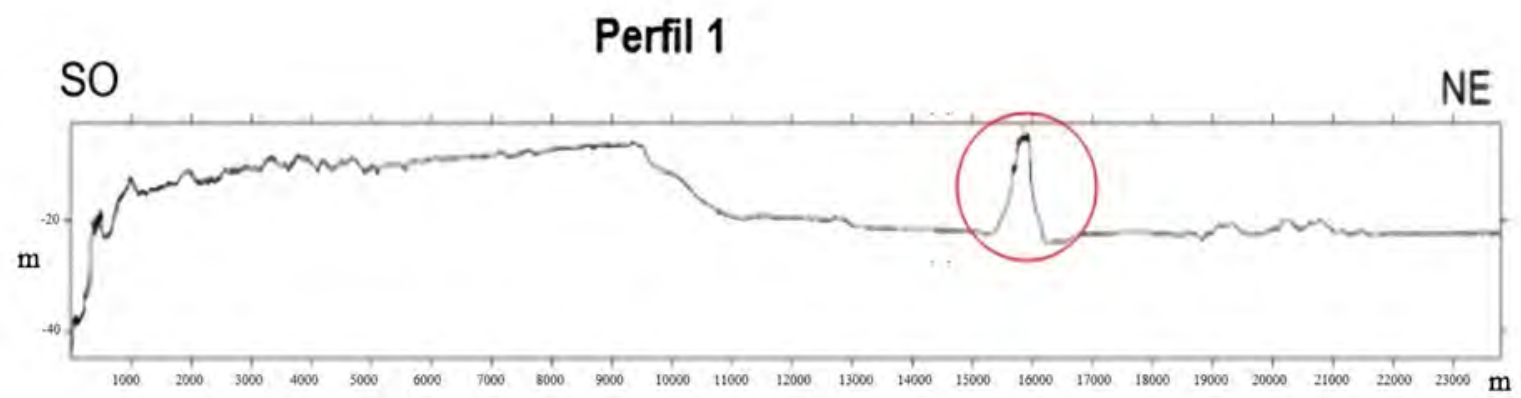

Perfil 2

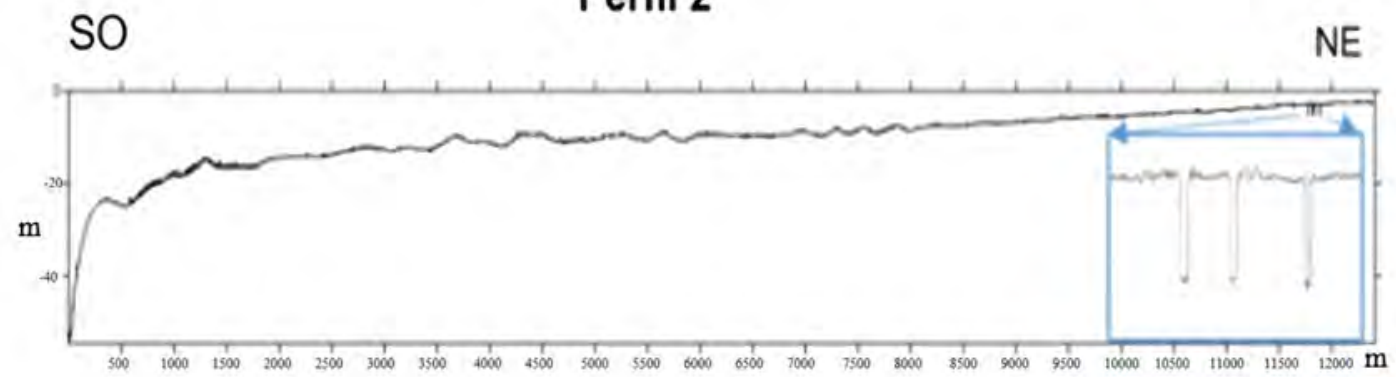

Figura 6. Resultados en perfiles batimétricos. Perfil 1 muestra un posible volcán de lodo (señalado con un círculo de color rojo). Perfil 2 en el que se identifican tres posibles pockmarks (señalados en el cuadro ampliado de color azul).

\section{Anomalías acústicas y morfologías asociadas a hidrocarburos}

En esta sección se caracterizan las anomalías acústicas y morfológicas más representativas que evidencian índices indirectos de manifestaciones de hidrocarburos en la Zona I, Zona II y en la Zona III (Figura 7).

\section{Zona I}

La anomalía A21_se caracteriza por una serie de depresiones del sub-fondo marino entre los 33,0 y 35,0 $\mathrm{m}$ de profundidad por debajo NMM, reflejando una sucesión vertical de las fases de la señal sísmica. Esta anomalía presenta gran semejanza con la descrita por Ercilla y Baraza (1996) en las investigaciones realizadas en el talud del Golfo de Cádiz, donde se presenta en un registro sísmico una serie de pockmarks superficiales (Figura 8).

\section{Zona II}

En la anomalía A37 se destaca la ruptura e irrupción de los reflectores sísmicos dentro de una unidad de facies sísmicas con reflectores continuos laterales. Esta intrusión dentro de los sedimentos marinos posee morfología en forma de cono con una altura de 20,0 m sobre la base del fondo marino, hasta una altura de 4,0 por debajo del NMM, como muestra el perfil batimétrico. Se destaca también la carencia de reflectores sísmicos por debajo de esta estructura, en una zona donde las facies sísmicas están caracterizadas por reflectores con continuidad lateral, configuraciones paralelas y varias secuencias sedimentarias (Figura 9).

\section{Zona III}

Las anomalías sísmicas y batimétricas A69, A70 y A72 presentan un comportamiento muy similar (Figura 10), determinadas por una irrupción de los reflectores sísmicos dentro de los sedimentos marinos, visualizándose la presencia de un patrón de reflexión caótico sobre la superficie del fondo marino y reflexiones difusas por debajo de este. La A72 presenta una altura de 7,0 $\mathrm{m}$ y un ancho de $60,0 \mathrm{~m}$ y la A70 tiene una altura de 5,0 $\mathrm{m}$ y ancho de 22,0 $\mathrm{m}$. Son estas estructuras típicas de volcanes de lodo registradas en otras latitudes. Es posible suponer que la A72 este asociada a un posible volcán de lodo más maduro, mientras que los A69 y A70 sean más recientes. 


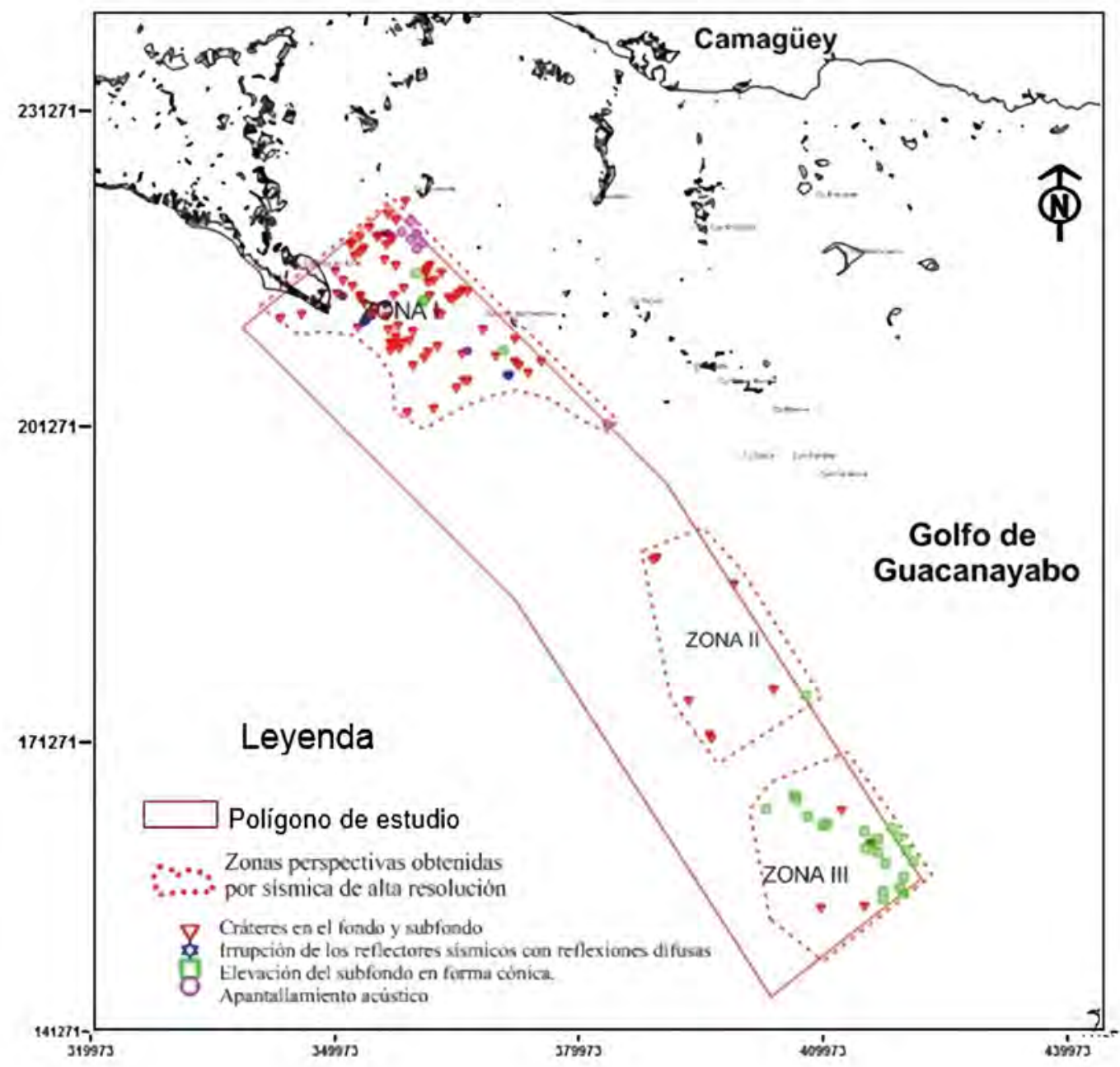

Figura 7. Clasificación de anomalías acústicas del subsuelo y geoformas del fondo marino asociadas a hidrocarburos en el área de estudio.

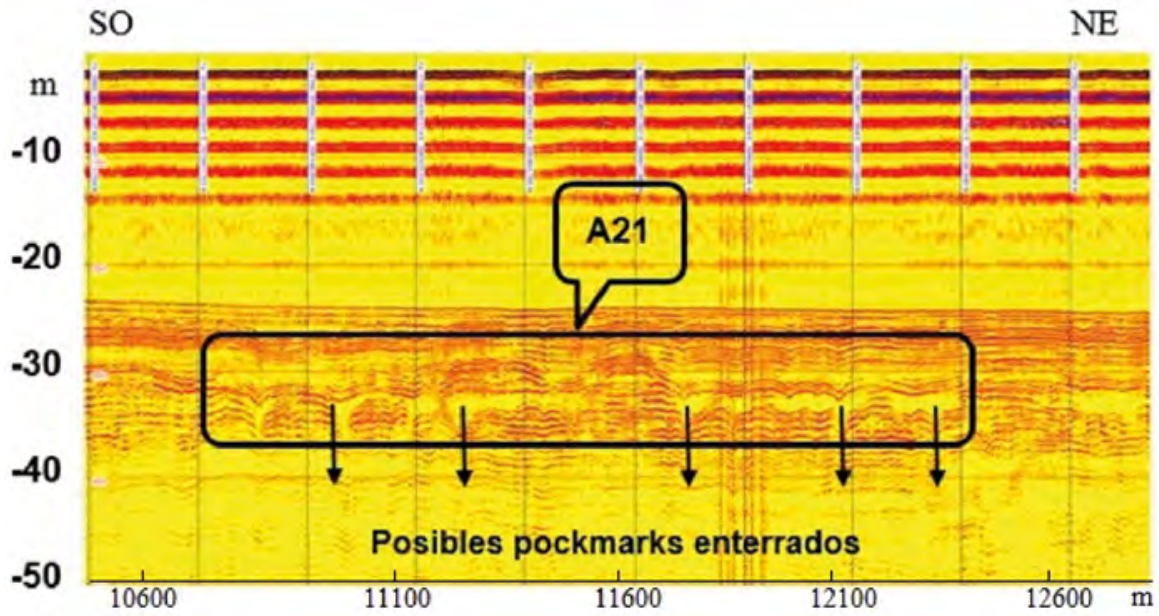

Figura 8. Registro sísmico en el Golfo de Guacanayabo donde se identifican posibles pockmarks enterrados (anomalía acústica A21). 


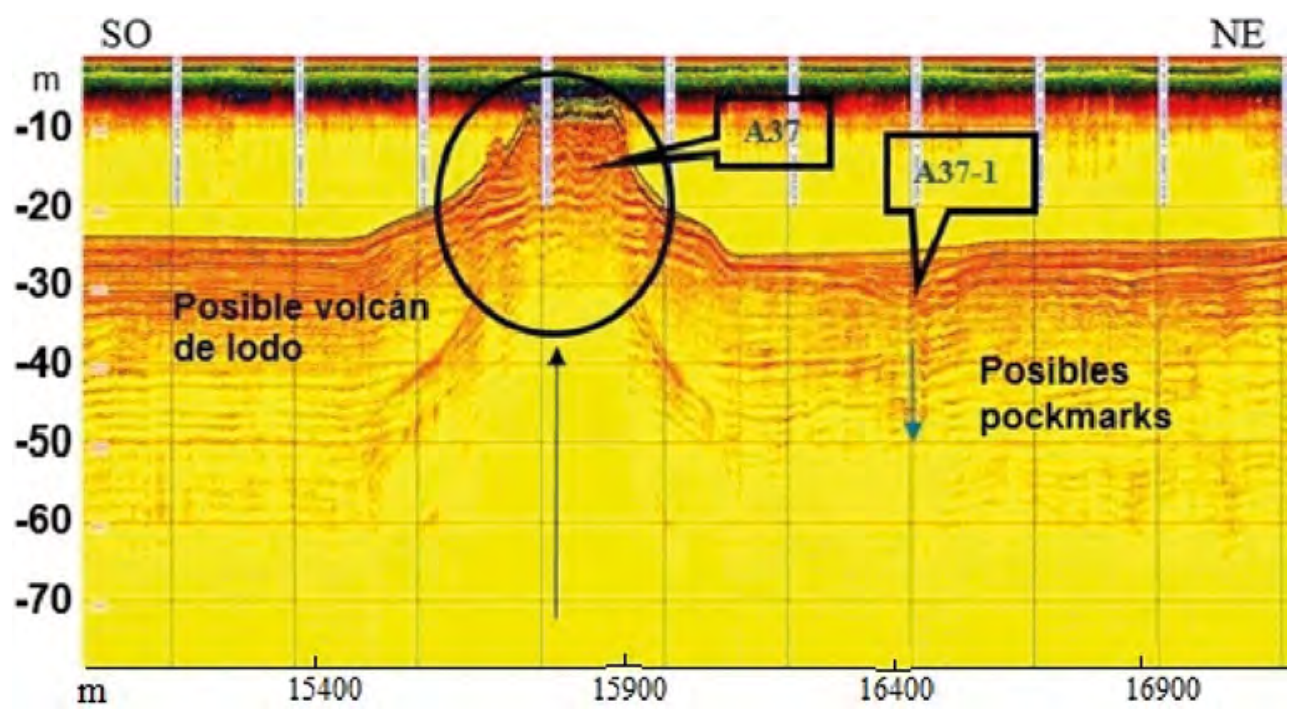

Figura 9. Registro sísmico en el Golfo de Guacanayabo donde se observan las anomalías A37 y A37-1.
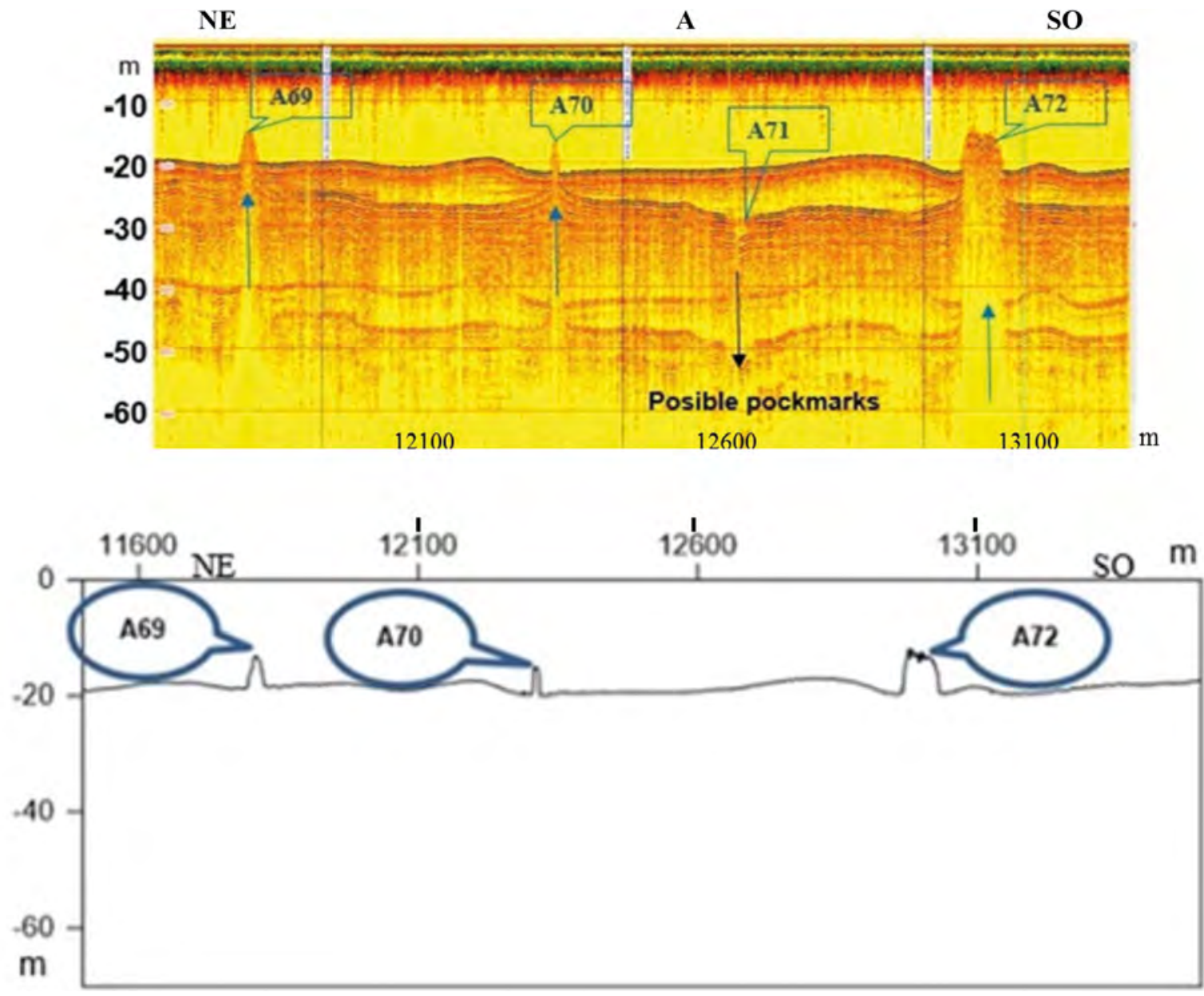

B

Figura 10. A. Registro sísmico del Golfo de Guacanayabo donde se observan las anomalías A69, A70, A71 y A72 interpretadas como posibles volcanes de lodo. B. Perfil batimétrico. 
La Figura 11 muestra la localización de 19 anomalías acústicas detectadas en el área, en las que se identifican posibles pockmarks, emanaciones gaseosas, volcanes de lodo y apantallamiento acústico, todas índices indirectos de manifestaciones de hidrocarburos.
Estas anomalías fueron cartografiadas en los registros sísmicos hasta profundidades de $60 \mathrm{~m}$, pudiendo ser correlacionadas con las estructuras profundas referidas en la zona por otros investigadores.

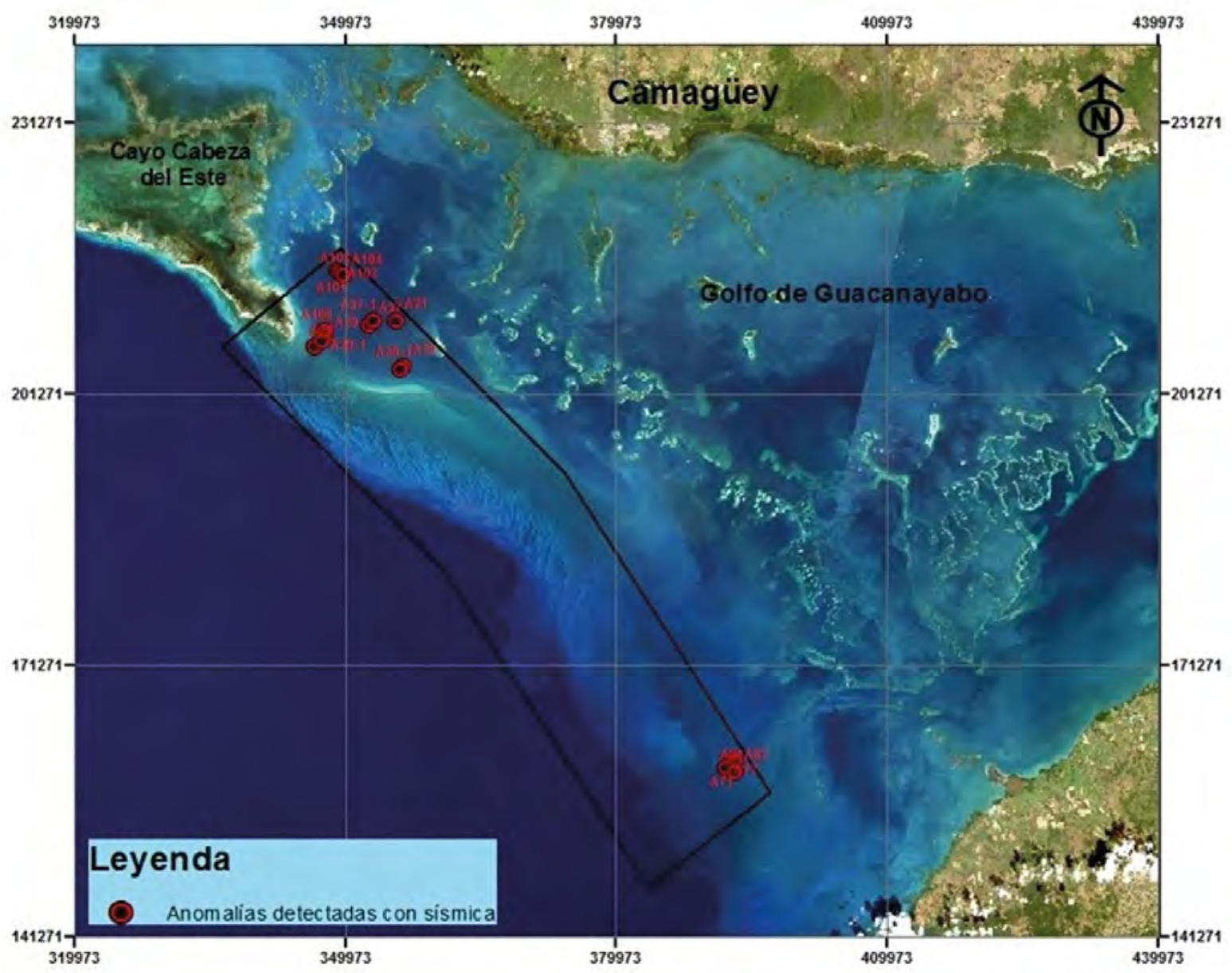

Figura 11. Anomalías acústicas detectadas con sísmica de alta resolución.

\section{Discusión}

El procesamiento de los datos batimétricos permitió detallar la superficie del fondo marino como límite superior de las secuencias sedimentarias. La misma muestra un comportamiento bastante irregular con profundidades que varían entre 0,0 y 40,0 m aproximadamente.

Hacia el sector sur del polígono de estudio se encuentra la presencia del talud insular, el cual declina de forma abrupta hasta profundidades entre 500 y $1000 \mathrm{~m}$.

La parte más profunda de la depresión Guacanayabo constituye la zona de la laguna interior donde se depositó el mayor volumen de los sedimentos terrígenos acarreados por la cuenca fluvial del río Cauto. En esta depresión fueron detectadas la mayor cantidad de anomalías acústicas dentro de las secuencias sedimentarias, lo que la clasifica como la más favorable para la presencia de petróleo y gas dentro de los sedimentos marinos. Aquí los fondos marinos se caracterizan por presentar una morfología plana con profundidades que oscilan alrededor de los $25 \mathrm{~m}$ y que disminuyen con pendientes bruscas hacia los bajos y cayos existentes en el área. Los espesores de sedimentos friables en el sector de estudio están en el orden de los 3 a $5 \mathrm{~m}$, por debajo de los mismos se detectaron varias fronteras sísmicas que son reflejo de la deposición de diferentes secuencias sedimentarias. 
En los registros sísmicos de alta resolución se detectaron 19 anomalías acústicas, agrupadas en tres zonas de interés por su grado de amplitud y características específicas. Las zonas I y III presentan anomalías y estructuras sismo-geológicas bien definidas, por lo que se consideraron las áreas más perspectivas desde el punto de vista gaso-petrolífero.

Los resultados presentados en esta ocasión muestran anomalías similares a las de otros investigadores en el ámbito internacional (Ercilla y Baraza, 1996; García-
Gil et al., 2000; Betzler et al., 2011; Quintero-Ramírez, 2012).

Las zonas con alta densidad de anomalías acústicas se han comparado con zonas perspectivas determinadas por la sísmica profunda 2D (Figura 12). Las zonas de perspectivas profundas coinciden con zonas de mayor densidad en anomalías acústicas del subsuelo identificadas con la sísmica de alta resolución (Morales-Carrillo et al., 2014; Álvarez-Ortiz et al. 2017; Morales-Carrillo, 2019).

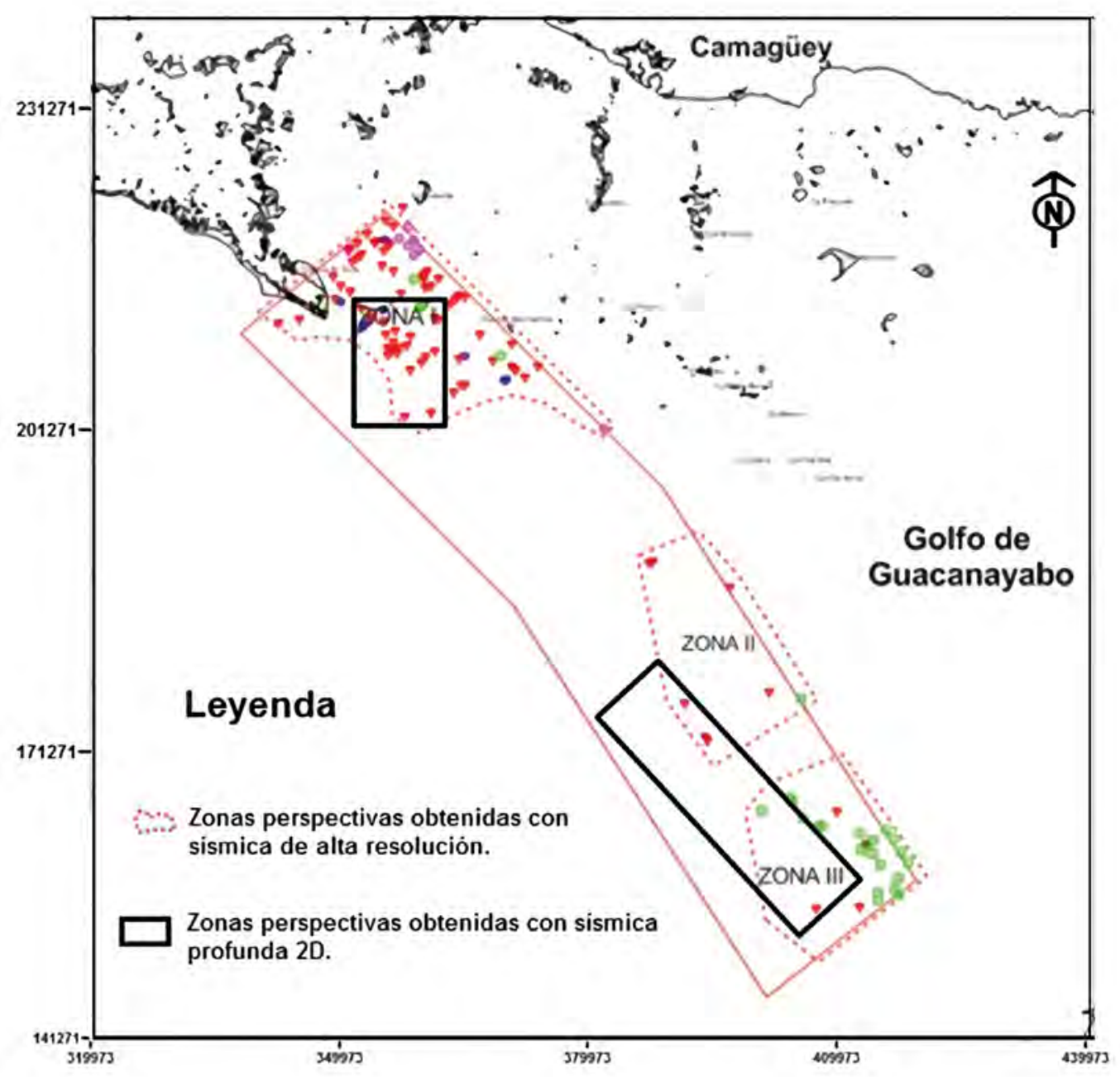

Figura 12. Zonas perspectivas determinadas por sísmica profunda y somera en el Golfo de Guacanayabo. Área de estudio señalada por el polígono en color rojo. 


\section{Conclusiones}

La interpretación conjunta de datos batimétricos y de perfiles sísmicos de reflexión de alta resolución ha permitido cartografiar estructuras que representan índices indirectos de manifestaciones de hidrocarburos en el fondo marino del Golfo de Guacanayabo.

Se han identificado tres tipos de formas y anomalías i) formas cónicas irrumpiendo los reflectores interpretados como posibles volcanes de lodo; ii) cráteres interpretados como emisiones de gas o pockmarks, y iii) anomalías acústicas del subsuelo relacionadas con la presencia de gas en los sedimentos similares a las obtenidas en otras regiones del mundo. La mayor densidad de estas anomalías acústicas se localiza en la parte más profunda de la depresión Guacanayabo que constituye la zona de la laguna interior, donde se depositó el mayor volumen de los sedimentos terrígenos acarreados por la cuenca fluvial del río Cauto.

Las áreas más perspectivas, desde el punto de vista gaso-petrolífero, se localizaron en las regiones donde coinciden las anomalías detectadas en los registros sísmicos de alta resolución y las detectadas por la sísmica profunda 2D, es decir donde existe una estrecha correlación entre las morfo-estructuras profundas y someras.

Se recomienda emplear este procedimiento de trabajo en otras zonas de la plataforma de Cuba y del Caribe, con el fin de cartografiar estos índices indirectos de la presencia de hidrocarburos, así como incorporar la interpretación de imágenes satelitales para la identificación de estructuras asociadas a los pockmarks $\mathrm{y}$ volcanes de lodo.

\section{Agradecimientos}

Los autores desean reconocer el apoyo brindado por la dirección de la empresa GEOCUBA, EM durante la realización de la adquisición de los materiales primarios utilizados en esta oportunidad, en especial al Ingeniero Geofísico Jorge Luis Perdomo Castillo. Además, reconocer la participación de la Ingeniera Geofísica Isabel Morales Castillo en los estadios iniciales de la investigación. Finalmente, agradecer a la Dra. Ing. Rosa María Valcarce Ortega del Departamento de Geociencias, de la Universidad Tecnológica de La Habana “José Antonio Echeverría”, por su revisión y corrección del documento original.

\section{Referencias}

Álvarez-Castro, J.; Valladares-Amaro, S.; PérezMartínez, Y.; Rodríguez-Menduiña, R. (2007). Metodología a aplicar en la fase de conclusión de la exploración en prospectos cubanos. Casos de la FNCP. II Convención Cubana de Ciencias de la Tierra. I Congreso de Petróleo y Gas. La Habana, Cuba.

Álvarez-Ortiz, M.; Perdomo-Castillo, J.L.; ProBetancourt, J.L.; Hernández-Valdés, O.; Veliz-Basabe, J.M.; Miró-Pagés, G. (2007). Consideraciones de la reinterpretación de datos geofísicos en la Bahía de Cárdenas, referentes a la exploración petrolera. Provincia de Matanzas. II Convención Cubana de Ciencias de la Tierra. III Congreso de Geofísica. La Habana, Cuba.

Álvarez-Ortiz, M.; Morales-Carrillo, I.; PerdomoCastillo, J.L. (2017). Detección con sísmica de alta resolución de evidencias de manifestaciones de hidrocarburos al sur del Golfo de Guacanayabo. VII Convención Cubana de Ciencias de la Tierra. IX Congreso de Geofísica. La Habana, Cuba.

Audsley, A.; Bradwell, T.; Howe, J.A.; Baxter, J.M. (2019). Distribution and classification of pockmarks on the seabed around western Scotland. Journal of Maps, 15(2), 807-817. https://doi.org/1 $0.1080 / 17445647.2019 .1676320$

Betzler, C.; Lindhorst, S.; Hübscher, C.; Lüdmann, T.; Fürstenau, J.; Reijmer, J. (2011). Giant pockmarks in a carbonate platform (Maldives, Indian Ocean). Marine Geology, 289(1-4), 1-16. https://doi. org/10.1016/j.margeo.2011.09.004

Ceramicola, S.; Dupré, S.; Somoza, L.; Woodside, J. (2018). Cold seep systems. In: A. Micallef, S. Krastel, A. Savini (eds.). Submarine geomorphology (pp. 367-387). Springer. https:// doi.org/10.1007/978-3-319-57852-1_19

Ercilla, G.; Baraza, J. (1996). Evidencias de gas en sedimentos del talud del Golfo de Cádiz. Geogaceta, 20(1), 180-182.

García-Gil, S.; García-García, A.; Vilas, F. (1999). Identificación sísmico-acústico de las diferentes formas de aparición de gas en La Ría de Vigo (NO de España). Revista Sociedad Geológica de España, 12(2), 301-307. 
García-Gil, S.; García-García, A.; Durán, R.; Vilas, F. (2000). Estratigrafía sísmica de alta resolución en las Rías Baixas: Pontevedra y Vigo (NO España). Journal of Iberian Geology, 26, 217-231.

Gay, A.; Lopez, M.; Cochonat, P.; Séranne, M.; Levaché, D.; Sermondadaz, G. (2006). Isolated seafloor pockmarks linked to BSRs, fluid chimneys, polygonal faults and stacked Oligocene-Miocene turbiditic palaeochannels in the Lower Congo Basin. Marine Geology, 226(1-2), 25-40. https:// doi.org/10.1016/j.margeo.2005.09.018

Hovland, M.; Gardner, J.V.; Judd, A.G. (2002). The significance of pockmarks to understanding fluid flow processes and geohazards. Geofluids, 2(2), 127-136. https://doi.org/10.1046/j.14688123.2002.00028.x

Iturralde-Vinent, M.A.; García-Casco, A. (2011). Compendio de Geología de Cuba y del Caribe. Segunda Edición, CITMATEL.

King, L.H.; MacLean, B. (1970). Pockmarks on the Scotian Shelf. GSA Bulletin, 81(10), 3141-3148. https://doi.org/10.1130/00167606(1970)81[3141:POTSS]2.0.CO;2

Krämer, K.; Holler, P.; Herbst, G.; Bratek, A.; Ahmerkamp, S.; Neumann, A.; Bartholomä, A.; van Beusekom, J.E.; Holtappels, M.; Winter, C. (2017). Abrupt emergence of a large pockmark field in the German Bight, southeastern North Sea. Scientific Reports, 7. https://doi.org/10.1038/ s41598-017-05536-1

León, R.; Somoza, L.; Medialdea, T.; HernándezMolina, F.J.; Vázquez, J.T.; Díaz-del-Río, V.; González, F.J. (2010). Pockmarks, collapses and blind valleys in the Gulf of Cádiz. Geo-Marine Letters, 30(3-4), 231-247. https://doi.org/10.1007/ s00367-009-0169-z

Leyva-Ramos, E. (2017). Cartografía de estructuras geológicas que evidencien índices de manifestaciones de hidrocarburos en el Golfo de Guacanayabo. Tesis, Universidad Tecnológica de La Habana “José Antonio Echeverría”, La Habana, Cuba.
Morales-Carrillo, I.; Álvarez-Castro, J.; GonzálezCaraballo, R.; Miró-Pagés, G. (2014). Alternativas para la exploración petrolera en los mares al sur de Cuba. Revista Cubana de Ingeniería, 5(1), 1925.

Morales-Carrillo, I. (2019). Anomalías sísmicas de interés para la exploración de hidrocarburos en el Golfo de Guacanayabo. VIII Convención de Ciencias de la Tierra. VII Congreso de Petróleo y Gas. La Habana, Cuba.

Quintero-Ramírez, J.D. (2012). Interpretación sísmica de volcanes de lodo en la zona occidental del abanico del delta del río Magdalena, Caribe colombiano. Tesis, Universidad EAFIT, Medellin, Colombia.

Rejas-Alejos, M. (2009). Génesis de carbonatos autigénicos asociados a volcanes de fango del Golfo de Cádiz (SW España): influencia de procesos bioquímicos y desestabilización de hidratos de gas. Tesis Doctoral, Universidad de Barcelona, España.

Somoza, L.; Gardner, J.M.; Díaz-del-Río, V.; Vázquez, J.T.; Pinheiro, L.M.; HernándezMolina, F.J.; TASYO/ANASTASYA shipboard scientific parties. (2002). Numerous methane gas-related sea floor structures identified in Gulf of Cadiz. Eos, Transactions American Geophysical Union, 83(47), 541-549. https://doi. org/10.1029/2002EO000371

Somoza, L.; Díaz-del-Río, V.; León, R.; Ivanov, M.; Fernández-Puga, M.C.; Gardner, J.M.; Hernández-Molina, F.L.; Pinheiro, L.M.; Rodero, J.; Lobato, A.; Maestro, A.; Vázquez, J.T.; Medialdea, T.; Fernández-Salas, L.M. (2003). Seabed morphology and hydrocarbon seepage in the Gulf of Cádiz mud volcano area: Acoustic imagery, multibeam and ultra-high resolution seismic data. Marine Geology, 195(1-4), 153-176. https://doi.org/10.1016/S0025-3227(02)00686-2

Somoza, L.; Medialdea, T.; León, R.; Ercilla, G.; Vázquez, J.T.; Farran, M.; Hernández-Molina, J.; González, J.; Juan, C.; Fernández-Puga, M.C. (2012). Structure of mud volcano systems and pockmarks in the region of the Ceuta Contourite 
Depositional System (Western Alborán Spina, R. (2018). Pockmarks as indicators to Sea). Marine Geology, 332-334, 4-26. https://doi. decipher some natural phenomena in the field of org/10.1016/j.margeo.2012.06.002

Spina, R. (2017). The pockmark stars: radial structures in the seabed surrounding the Hawaii Islands. Journal of Environmental Geology, 1(1), 33-50. geology and beyond: state of knowledge and its implications. Journal of Environmental Geology, 2(S1), 1-2.

Fecha de recibido: 26 de junio de 2020

Fecha de aprobado: 01 de marzo de 2021 